ARTICLE

DOI: $10.1038 / s 41467-018-04613-x$

\title{
Live cyanobacteria produce photocurrent and hydrogen using both the respiratory and photosynthetic systems
}

Gadiel Saper ${ }^{1}$, Dan Kallmann ${ }^{1}$, Felipe Conzuelo ${ }^{2}$, Fangyuan Zhao ${ }^{2}$, Tünde N. Tóth ${ }^{1,3}$, Varda Liveanu ${ }^{4}$, Sagit Meir ${ }^{5}$, Jedrzej Szymanski ${ }^{5,6}$, Asaph Aharoni ${ }^{5}$, Wolfgang Schuhmann (1) 2, Avner Rothschild ${ }^{1,7}$, Gadi Schuster $^{1,4}$ \& Noam Adir (iD) ${ }^{1,3}$

Oxygenic photosynthetic organisms perform solar energy conversion of water and $\mathrm{CO}_{2}$ to $\mathrm{O}_{2}$ and sugar at a broad range of wavelengths and light intensities. These cells also metabolize sugars using a respiratory system that functionally overlaps the photosynthetic apparatus. In this study, we describe the harvesting of photocurrent used for hydrogen production from live cyanobacteria. A non-harmful gentle physical treatment of the cyanobacterial cells enables light-driven electron transfer by an endogenous mediator to a graphite electrode in a biophotoelectrochemical cell, without the addition of sacrificial electron donors or acceptors. We show that the photocurrent is derived from photosystem I and that the electrons originate from carbohydrates digested by the respiratory system. Finally, the current is utilized for hydrogen evolution on the cathode at a bias of $0.65 \mathrm{~V}$. Taken together, we present a biophotoelectrochemical system where live cyanobacteria produce stable photocurrent that can generate hydrogen.

\footnotetext{
${ }^{1}$ The Nancy \& Stephen Grand Technion Energy Program (GTEP), Technion - Israel Institute of Technology, Technion City, 32000 Haifa, Israel. ${ }^{2}$ Analytical Chemistry - Center for Electrochemical Sciences (CES), Ruhr-Universität Bochum, Universitätsstr. 150, 44780 Bochum, Germany. ${ }^{3}$ Schulich Faculty of Chemistry, Technion - Israel Institute of Technology, Technion City, 32000 Haifa, Israel. ${ }^{4}$ Faculty of Biology, Technion - Israel Institute of Technology, Technion City, 32000 Haifa, Israel. ${ }^{5}$ Department of Plant and Environmental Sciences, The Weizmann Institute of Science, Rehovot, Israel. ${ }^{6}$ Leibniz Institute of Plant Genetics and Crop Research (IPK), Network Analysis and Modelling, OT Gatersleben, 06466 Seeland, Germany. ${ }^{7}$ Department of Materials Science and Engineering, Technion - Israel Institute of Technology, Technion City, 32000 Haifa, Israel. These authors contributed equally: Gadiel Saper, Dan Kallmann. Correspondence and requests for materials should be addressed to G.S. (email: gadis@technion.ac.il) or to N.A. (email: noam@ch.technion.ac.il)
} 
C yanobacteria are a wide class of photosynthetic organisms that have been recently proposed as potential sources of materials (membranes, complexes) from which photoexcited electrons can be extracted to produce electrical current and renewable fuels ${ }^{1-3}$. Different species of cyanobacteria living in a wide range of habitats, were responsible for the original formation of the Earth's oxygen enriched atmosphere, and continue to serve as a major source of primary production and oxygen evolution ${ }^{4}$. In cyanobacterial photosynthesis, the major photosynthetically relevant light absorbing pigment is chlorophyll (chl) $a$, which has maximal absorbance at $420-460 \mathrm{~nm}$ and 670-700 nm. The phycobilisome (PBS) antenna complex additionally absorbs between $550-680 \mathrm{~nm}$. Photosystem I (PSI) also contains a minor antennae complex that has been shown to be red-shifted to $\sim 710-730 \mathrm{~nm}^{5-8}$. The energy is efficiently transferred from the antennas to the Photosystem II (PSII) and PSI reaction centers $\left(\mathrm{P}_{680} \text { and } \mathrm{P}_{700} \text {, respectively }\right)^{9-11}$. In this lightdriven process, electrons from water are sequentially transferred through a series of redox species to finally reduce $\mathrm{NADP}^{+}$to be used for carbon fixation ${ }^{12,13}$ (Fig. 1). A proton-motive gradient is created between the thylakoid membrane lumen and the cytoplasm, resulting in proton-coupled electron transfer, which serves as the driving force for ATP production.

In the dark, cyanobacterial cells oxidize carbon sources via the respiratory system. For example, glucose is oxidized to acetyl CoA in the glycolytic process. Acetyl CoA is further oxidized in the tricarboxylic acid (TCA) cycle. These processes produce ATP, $\mathrm{NADH}$, and $\mathrm{FADH}_{2}$. NADH and $\mathrm{FADH}_{2}$ are oxidized during

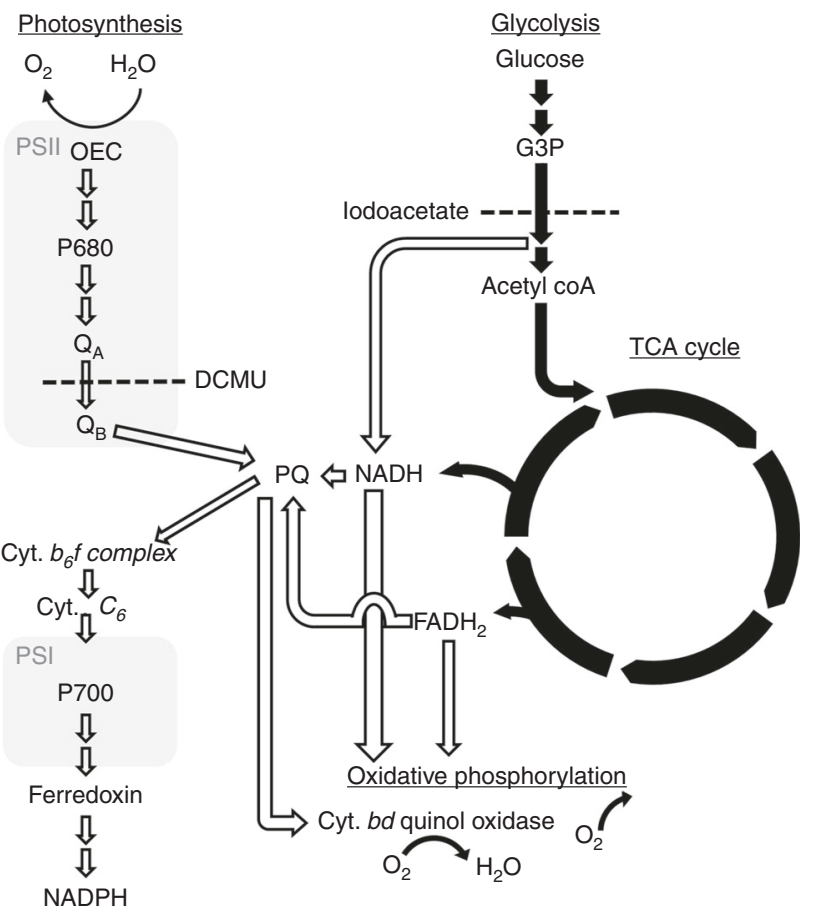

Fig. 1 The electron flow of the photosynthetic process and respiration metabolism in cyanobacteria. The scheme was drawn based on the data described in references $12-21$. Filled black arrows mark the respiration metabolism. Unfilled arrows indicate the photosynthetic and additional electron flow processes. Double arrows mark additional steps not shown for clarity. G3P is glyceraldehyde 3 phosphate, and TCA stands for tricarboxylic acid cycle. The membrane soluble plastoquinone (PQ) functions as an acceptor of residual electrons of the glycolysis and the TCA cycle, as well as an essential mediator of the photosynthetic electrons between PSII and the $b_{6} f$ complex. Dashed lines indicate the site of action of inhibitors of the photosynthetic or respiratory, used in this work oxidative phosphorylation to consume oxygen and produce $\mathrm{CO}_{2}$ and $\mathrm{ATP}^{14-16}$. Cyanobacteria lack the separation afforded by eukaryotic organelles, and it has been previously shown that electrons can flow between the photosynthetic and respiratory systems via redox active molecules ${ }^{17}$. For example, quinones from the photosynthetic plastoquinone (PQ) pool can oxidize $\mathrm{NADH}$ and $\mathrm{FADH}_{2}$, both products of glucose oxidation ${ }^{17-21}$ (Fig. 1).

Various attempts have been made to extract electrons from photosynthetic systems for the purpose of solar energy conversion (SEC) ${ }^{19,22-29}$. In some studies, isolated photosystems (PS) have been used within a bio-photoelectrochemical cell (BPEC) to produce electric power ${ }^{30-34}$. With isolated PS, an exogenous electron acceptor has to be used as a mediator (when utilizing PSI or bacterial reaction centers, an exogenous donor is also required) and the PS has to be fixed to the electrode, for instance by the presence of an immobilized mediator or a redox polymer $24,25,31,34$. In the case of PSII, water can serve as the electron donor, dependent on the stability of the oxygen evolving complex (OEC). One of the main problems with using isolated photosynthetic complexes for SEC is the relatively short lifetime of the biological components of the system, due to damage caused by radicals formed within the reaction centers ${ }^{35,36}$. Combined membrane systems have an evolutionary benefit for energy conversion that has recently been identified as a characteristic that could improve artificial photosynthetic SEC systems ${ }^{35}$. The use of living cells could mitigate this problem due to the presence of repair systems that can replace photo-damaged photosynthetic proteins. However, extracting electrons from living organisms is potentially more complicated as it may require an exogenous electron carrier (mediator) that can penetrate the cell wall and membrane, such as ferricyanide, cytochrome, or 2,6-dichloro-1,4benzoquinone (DCBQ) ${ }^{23,27,36-39}$. To overcome this difficulty, cyanobacterial cells have been grown or dried on an electrode to extract an electric current by direct contact ${ }^{1,28,40,41}$. However, when such direct contact is utilized, the electric current is limited, as it will likely be generated from only a single layer of cells.

Here, we describe the construction of a BPEC in which live cyanobacteria produce a significant photocurrent that is derived from electrons of the respiratory and photosynthetic systems, without the need for exogenous electron donors or mediators. The resulting current was utilized for the production of molecular hydrogen on the cathode at a bias voltage of $0.65 \mathrm{~V}$.

\section{Results and Discussion}

Production of a significant current by living cells. Cyanobacterial cells, Synechocystis sp. PCC 6803, were gently treated with a microfluidizer (herein called iSyn, see methods), and applied by gravity onto the graphite electrode. A platinum wire serves as the cathode while an $\mathrm{Ag} / \mathrm{AgCl} / 3 \mathrm{M} \mathrm{NaCl}$ electrode is used as a reference in all measurements (BPEC, Fig. 2a). When we measured current over time (chronoamperometry, CA), at an applied potential of $150 \mathrm{mV}$ (vs. $\mathrm{Ag} / \mathrm{AgCl} / 3 \mathrm{M} \mathrm{NaCl}$ ) (standard working potential obtained from the IV curve (Supplementary Fig. 1A), for all CA experiments unless mentioned otherwise), a dark current of $5 \pm 1 \mu \mathrm{A} \mathrm{cm}^{-2}$ was obtained, probably directly from the respiratory system, as previously suggested ${ }^{36,37}$ (Fig. 2b, initial 100 seconds). Upon illumination, the current increased to the maximum stable current of $36 \pm 4 \mu \mathrm{A} \mathrm{cm}^{-2}$. Accordingly, the net produced photocurrent was $31 \pm 4 \mu \mathrm{A} \mathrm{cm}^{-2}$. Upon return to dark, there was a slow decline in the measured current. We noticed that the addition of the photosynthetic electron flow inhibitor, 3-(3,4-dichlorophenyl)-1,1-dimethylurea (DCMU), resulted in a significantly faster increase in the photocurrent, reaching the maximum value in about $3 \mathrm{~min}$, as compared to about $13 \mathrm{~min}$ in the absence of DCMU (Fig. 2b). Thus, DCMU 
a

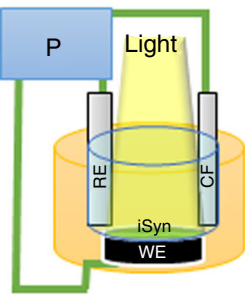

b

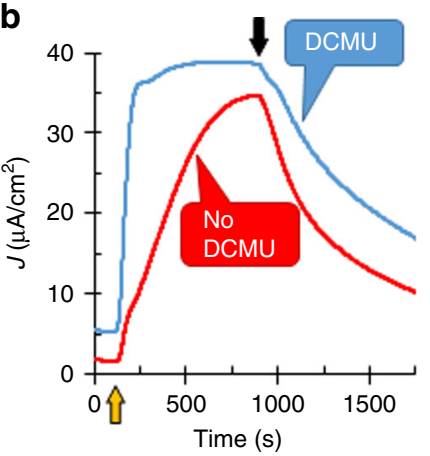

C

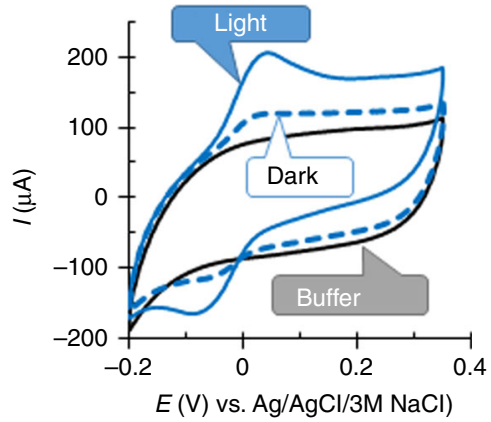

Fig. 2 Gently treated Synechocystis cells generate an enhanced photocurrent using an endogenous redox mediator. a Schematic drawing of the bio photoelectrochemical apparatus with the iSyn settled on the working electrode (WE). The counter electrode (CE) is platinum, and the reference electrode (RE) an $\mathrm{Ag} / \mathrm{AgCl} / 3 \mathrm{M} \mathrm{NaCl}$. All electrodes are connected to the potentiostat ( $\mathrm{P}$ ). b Chronoamperometric measurements at $150 \mathrm{mV}$ (vs. $\mathrm{Ag} / \mathrm{AgCl} / 3 \mathrm{M} \mathrm{NaCl}$ ) for iSyn in the presence (blue) or absence of DCMU (red). The yellow up arrow indicates light on (the initial 100 seconds show the dark current) and the black down arrow indicates light off. c Cyclic voltammograms of iSyn, illuminated (solid blue lines) or in the dark (dashed blue lines). Buffer lacking iSyn shows no redox activity (black solid line). Measurements recorded at a rate of $5 \mathrm{mV} \mathrm{s}^{-1}$
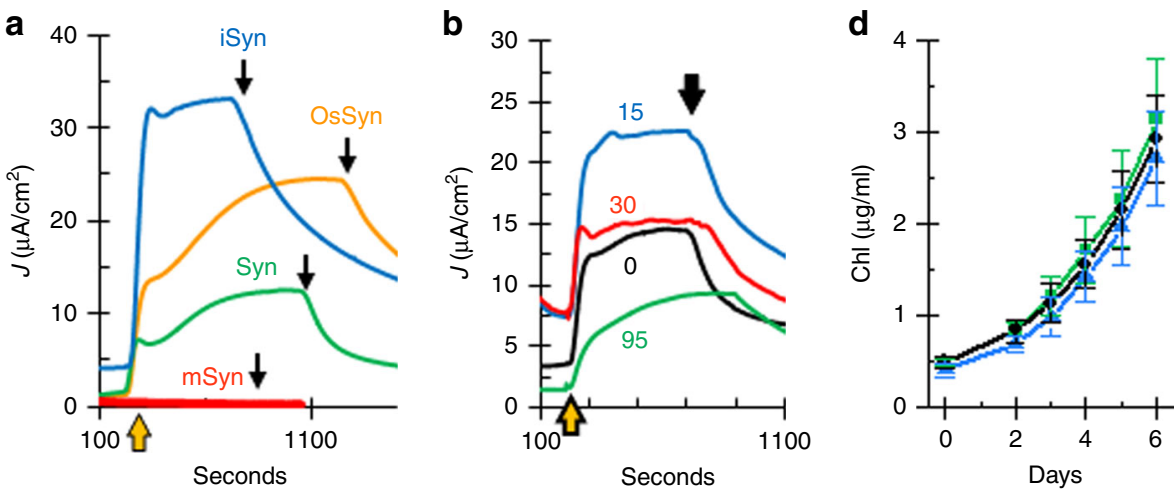

C
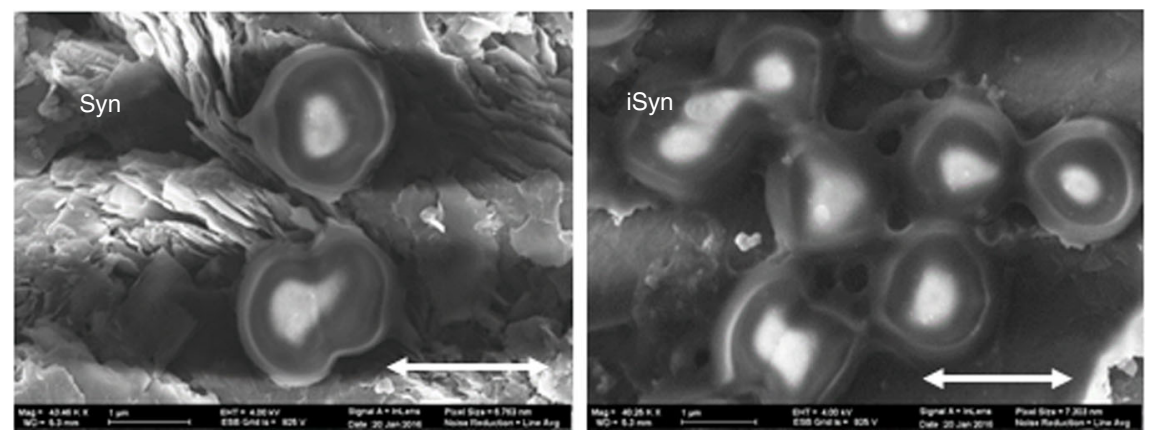

Fig. 3 iSyn are living, modified, and clustered cells. a CA measurements of Synechocystis cells (Syn, green), cells undergone a mild osmotic shock treatment, (OsSyn, Orange), cells undergone a gentle pressure treatment (iSyn, blue), and thylakoid membranes (mSyn, red). b CA measurements of cell undergone treatment with microfluidizer at 0, 15, 30, and 95 psi, as indicated in the figure. c SEM image of iSyn (right) and Syn (left) on graphite. Scale bars are $2 \mu \mathrm{m}$. d Growth curve of Syn (green), iSyn (black) and iSyn that were illuminated for $30 \mathrm{~min}$ in the BEPC (blue) in liquid medium. The vertical lines show the standard deviation $(n=5)$

was used for all electrochemical measurements unless mentioned otherwise. Cyclic voltammetry (CV) of the iSyn indicated the presence of a redox active species with an anodic peak at $50 \mathrm{mV}$ (vs. $\mathrm{Ag} / \mathrm{AgCl} / 3 \mathrm{M} \mathrm{NaCl}$ ) and a cathodic peak at $-70 \mathrm{mV}$ (vs. $\mathrm{Ag} /$ $\mathrm{AgCl} / 3 \mathrm{M} \mathrm{NaCl}$ ). The anodic current was significantly increased during illumination to present a quasi-reversible voltammogram, suggesting a photo-induced catalytic reaction mediated by a redox species (Fig. 2c). Optimization experiments show that increasing the amount of cells increases both CV (Supplementary Fig. 1B) and CA (Supplementary Fig. 1C) values up to a plateau of 150-225 $\mu \mathrm{g}$ chlorophyll. Since no external mediating electron transfer molecules were added to the BPEC, we conclude that the source of the redox species identified in Fig. $2 \mathrm{c}$ is from within the Syn cells. The characterization of this mediator will be described in detail below.

We noticed that there was a negative correlation between the pressure used during the treatment performed on the cells and the measured current (Fig. 3a). The highest current was obtained when the lowest possible pressure of the microfluidizer was used (Fig. 3b). Lower currents were obtained when using untreated cells (Syn) or cells that were osmotically shocked (OsSyn). Membrane fragments obtained from cells that were completely disrupted at higher pressure with a French Pressure cell (mSyn) did not produce measureable currents (Fig. 3a). The 

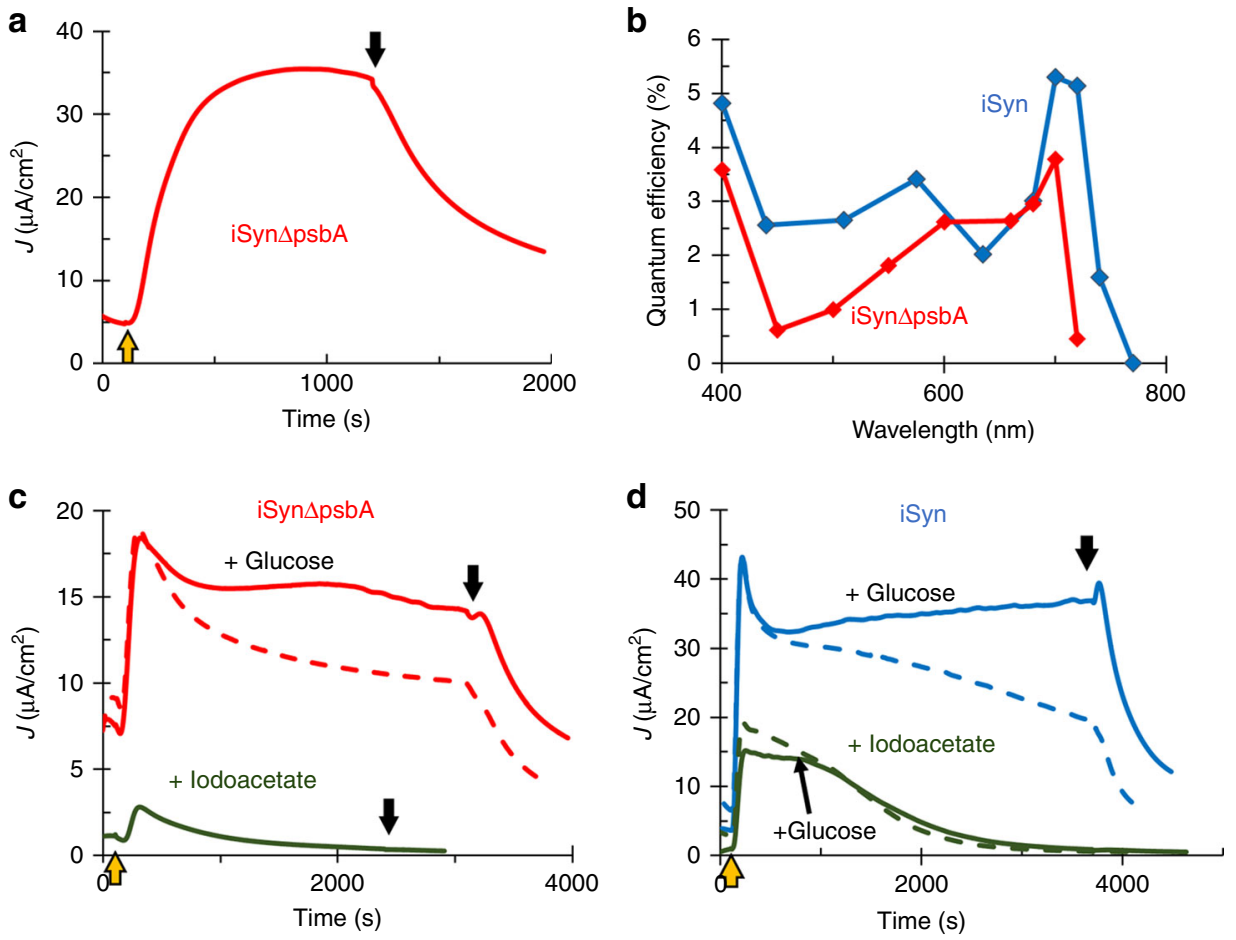

Fig. 4 iSyn photocurrent to the BPEC involves both photosynthetic and respiratory systems. a CA measurements for iSyn $\Delta$ psbA. b Action spectra: the obtained photocurrent was normalized to the photon energy input (external quantum efficiency) at different wavelengths. The external quantum efficiency of iSyn is shown in blue and that of iSyn $\Delta \mathrm{psbA}$ in red. A new measurement with fresh cells was used for each wavelength. c CA measurements for iSyn $\Delta \mathrm{psbA}$ without (red) and with (green) the addition of iodoacetate and in the presence (full line) or absence (dashed line) of glucose. $\mathbf{d}$ CA measurements for iSyn without (blue) and with (green) the addition of iodoacetate or in the presence (full line) or absence (dashed line) of glucose in the buffer

microfluidizer treatment (iSyn) resulted with a higher anodic peak in the dark, compared to OsSyn and Syn (Supplementary Fig. 1D), whereas membrane fragments did not show any anodic peak (Supplementary Fig. 1E) indicating the absence of the redox species (see methods for all preparations). iSyn appear to retain their cellular structure and remain clumped together as visualized by scanning electron microscopy (SEM) and florescent microscopy (Fig. 3c, Supplementary Fig. 2A, respectively). To further investigate the state of the iSyn preparation, we utilized confocal fluorescence microscopy. Syn, OsSyn, and iSyn appeared in the microscope to retain PBS functional integrity. Treatment of the same cells using higher pressures (mSyn) produces aggregated membranous material, lacking the PBSs (Supplementary Fig. 2A$\mathrm{C}$ ), which are soluble and spontaneously released from the membranes ${ }^{9}$. The major difference between the Syn and iSyn is that the latter are clumped together in large clusters (Fig. 3c, Supplementary Fig. 2A). In the OsSyn preparation, cells are seen to form smaller clusters of two or three cells with the same appearance of fusion (Supplementary Fig. 2A). To determined cell viability, we prepared cells as described above, and illuminated them for $30 \mathrm{~min}$ in the BPEC under standard conditions. We then removed the iSyn from the BPEC by simple washing. The growth rate of the iSyn was then compared to the growth rates of either untreated Syn or non-illuminated iSyn in new growth media. All cells had similar growth rates (Fig. 3d and Supplementary Fig. 3a). To further determine cell viability, we performed a colony formation test (Supplementary Fig. 3B). We applied an amount of Syn, iSyn, or membranes (mSyn) containing the same amount of chlorophyll onto a growth plate, and followed the growth of the colonies. Syn and iSyn grow at a similar rate, $364 \pm 98$ and $280 \pm 68$ colonies per femtograms of Chl $a$ to Syn and iSyn, respectively, whereas mSyn contains much smaller amount of living cells $(11 \pm 3)$. These results indicate that the application of mild pressure did not harm the ability of Synechocystis cells to multiply and divide. iSyn were also shown to photosynthetically evolve $\mathrm{O}_{2}$ at a similar rate to Syn cells $\left(\mathrm{O}_{2}\right.$ evolution of $392 \pm 53,464 \pm 60 \mathrm{nmol} \mathrm{O}_{2} \mu \mathrm{g} \mathrm{chl}^{-1} \mathrm{~h}^{-1}$ for iSyn and Syn, respectively). We thus suggest that iSyn are living, yet modified and/or clustered cells and that the change that occurs to the cells does not modify the biological energy transducing systems.

The combined activity of two pathways generates the current. The photocurrent in the presence of DCMU could be explained by two alternative pathways. In the first one, the electrons are derived from water oxidation and are transferred to putative endogenous mediator(s) prior to $\mathrm{Q}_{\mathrm{B}}$, before the binding site of DCMU at $\mathrm{Q}_{\mathrm{B}}$, similar to the electron transfer from PSII to silicomolybdate $^{42,43}$. The second possibility is that DCMU inhibits the activity of PSII, including the water oxidation complex. In such a case, a light-driven electron source must be located further downstream of the $\mathrm{Q}_{\mathrm{B}}$ site, and could involve the PQ pool, cytochrome $b_{6}$ f, and finally PSI, as the site of photoactivity. A stable photocurrent that is not a result of PSII activity can only exist if there is an alternative source of electrons to PSI. Such a possible source could be the respiratory system that is linked to the photosynthetic apparatus in cyanobacteria ${ }^{14,17-21}$ (Fig. 1). To differentiate between these possibilities we repeated the electrochemical experiments with a non-autotrophic mutant of Synechocystis sp. PCC 6803, where the psbA genes (encoding the D1 protein) have been deleted, thus leading to a non-functional PSII $(\triangle \mathrm{psbA})^{43,44}$ (Supplementary Fig. 4A). CA measurements displayed a photocurrent that is similar to that obtained with iSyn (Fig. 4a). Moreover, the iSyn $\Delta$ psbA cells look similar to iSyn cells (Supplementary Fig. 4B) and require the same preparation for 
a

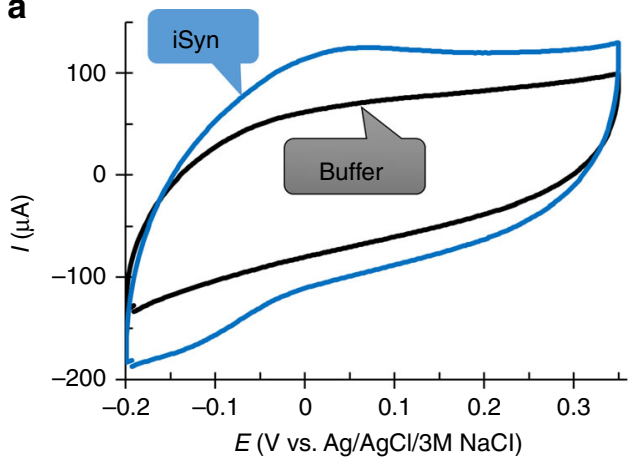

C

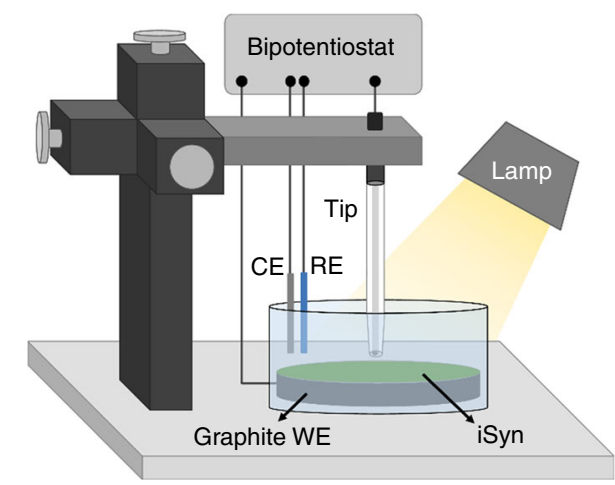

b

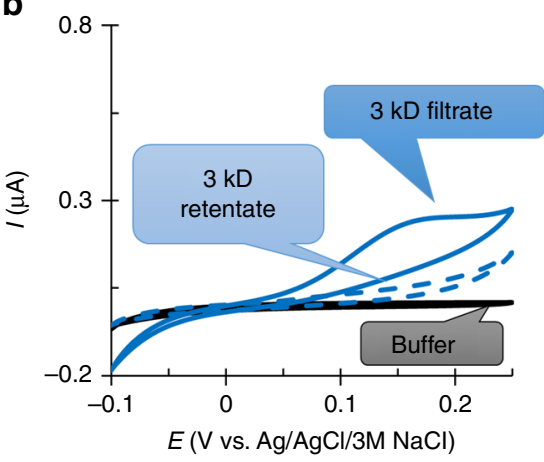

d

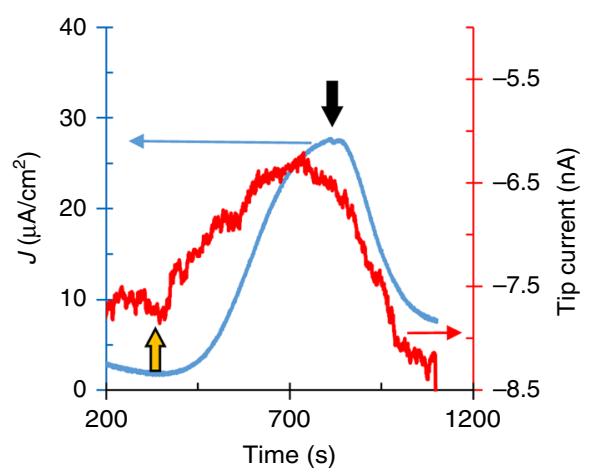

Fig. 5 iSyn electron transfer to the BPEC is via a diffusive endogenous mediator. a CV in the light for buffer (black) and the iSyn (blue) separated by a $3 \mathrm{kD}$ dialysis membrane on the graphite electrode. $\mathbf{b} \mathrm{CV}$ of supernatant fraction from centrifuged iSyn cells separated by filtration through $3 \mathrm{kD}$ cut-off centricon. Filtrate (solid blue line), retentate (dashed blue), and buffer (black). c Illustration of the scanning electrochemical microscopy setup. iSyn settled on the working electrode (WE) electrode. The counter electrode (CE) is platinum, and the reference electrode (RE) a $\mathrm{Ag} / \mathrm{AgCl} / 3 \mathrm{M} \mathrm{KCl}$. The tip is a carbon-based microelectrode with a mixture of bilirubin oxidase (BOD) embedded in an Os-complex modified polymer matrix. All electrodes are connected to the bipotentiostat. $\mathbf{d}$ CA of the iSyn (blue) and the BOD tip current (red) measuring the oxidation of the mediator at a distance of $30 \mu \mathrm{m}$ from the graphite electrode

optimal photocurrent as iSyn does (Supplementary Fig. 4B-D). The iSyn $\Delta$ psbA cells also contain the same redox mediator (Supplementary Fig. 4D). Another proof of the lack of involvement of PSII in the electron transfer pathway in the BPEC was to inactivate the OEC of PSII by incubation with Tris buffer. In the Tris treated iSyn similar photocurrents were obtained (Supplementary Fig. 5A-B). Taken together these results indicate that the electron path is from the respiratory system, through PSI and to the electrode.

To further support the notion that the photosystem deriving the photocurrent is PSI and not PSII, we measured the action spectrum of the photocurrent production. To this end, we measured the photocurrent as a function of the wavelength and calculated the external quantum efficiency (EQE) at each wavelength (Fig. 4b). The EQE in both iSyn and iSyn $\Delta$ psbA was evident at all wavelengths of visible light with an increase at $700 \mathrm{~nm}$ suggesting the importance of the participation of PSI in the activity. Together, these results demonstrated that PSI and not PSII is the photosystem that drives the photocurrent when iSyn cells are illuminated in the BPEC.

As already indicated above, the lack of the water oxidation activity of PSII in the iSyn $\Delta$ psbA indicates that there must be an alternative electron donor. Studies suggest that electron transfer between the respiratory system and the photosynthetic system may occur via the plastoquinone (PQ) pool ${ }^{14,18}$. To investigate this, we performed experiments in the presence of glucose. Glucose is known to be uptaken by Synechocystis cells, providing energy for growth in the dark or for growth of nonphotosynthetic mutants ${ }^{43,44}$. Addition of glucose significantly extended the period of the production of the highest current (Fig. 4c). Furthermore, iodoacetate, which blocks the respiratory path at the site of glyceraldehyde-3-phosphosphate dehydrogenase activity (Fig. 1), significantly inhibited the photocurrent (Fig. 4c), supporting the suggestion that the respiratory system is the main electron donor.

The above measurements support our hypothesis that under these conditions a respiratory-PSI electron transfer pathway exists in the $\triangle$ psbA mutant. In a fashion similar to that seen for iSyn $\Delta$ psbA, iSyn also exhibits extended photocurrent lifetime in the presence of glucose (Fig. $4 \mathrm{~d}$ and Supplementary Fig. 5C-D), and the photocurrent is decreased in the presence of iodoacetate (Fig. 4d), reaffirming that the major electron source is the respiratory system. Together, the results so far uncover an electron transfer pathway in which electrons derived from the respiratory pathway reduce PSI that can further photo-reduce the electrode in the gently treated Synechocystis live cells.

The mediator is a small diffusive soluble molecule. Electrochemical experiments show a slow increase and slower decline in the photocurrent (Fig. 2b). Moreover, the potential difference between the anodic and cathodic peaks in the voltammogram (Fig. 2c) suggests that the current in the BPEC is mediated by a diffusive soluble molecule. Cyanobacterial cells contain both small redox active proteins, as well as metabolites that undergo redox reactions. We introduced a single layer synthetic membrane with a nominal molecular weight cut-off of $3 \mathrm{kD}$ between the iSyn and the electrode (Fig. 5a) and performed CV 
measurements under light. These show the same anodic and cathodic peaks in the presence of iSyn as without the membrane, suggesting the mediator can diffuse through a $3-\mathrm{kD}$ membrane and is thus most likely not a protein.

To further isolate the mediator, iSyn were illuminated followed by centrifugation. The supernatant was then filtered through a 3 $\mathrm{kD}$ ultrafiltration device and CV measurements were performed on both the filtrate and retentate. These measurements show that only the filtrate contains a redox active species while the retentate has no redox active component (Fig. 5b). This indicates that the mediator is a water-soluble molecule that passes through the 3$\mathrm{kD}$ filter. Similar experiments performed on dark exposed iSyn (Supplementary Fig. 6A) showed the presence of a redox peak but with a smaller area, suggesting that illumination increases the release of the mediator from the cells. In addition, the redox behavior of the mediator is inactivated after $10 \mathrm{~min}$ incubation at $60{ }^{\circ} \mathrm{C}$ (Supplementary Fig. 6B). We suggest that the redox molecules could be a soluble quinone, a flavonoid ${ }^{44,45}$ or a small peptide that is temperature sensitive.

Scanning electrochemical microscopy (SECM) was used to exclude the possible release of $\mathrm{O}_{2}$ under irradiation and proved the existence of a soluble mediator in accordance with the CA measurements. SECM measures the local current on a microelectrode tip precisely positioned above the evaluated sample. SECM can be used to measure local electrochemical reactions over an evaluated sample or to efficiently collect reaction products with the tip placed in close proximity to the analyzed surface, for example, it was previously used to measure oxygen production by n-type semiconductor-based photo-anodes for water splitting ${ }^{45,46}$. The SECM experiment was performed by placing the tip microelectrode above the center of the cells at $\sim 30 \mu \mathrm{m}$ from the graphite electrode surface with iSyn deposited on top (Fig. 5c). As SECM probe we used a carbon-based microelectrode modified with bilirubin oxidase (BOD) embedded in an Os-complex modified polymer matrix ${ }^{47,48}$. The electrode was polarized at $0 \mathrm{mV}$ (vs. $\mathrm{Ag} / \mathrm{AgCl} / 3 \mathrm{M} \mathrm{KCl}$ ) for the steady-state reduction of $\mathrm{O}_{2}$. To decrease the background current related with the reduction of $\mathrm{O}_{2}$ naturally present in solution, Ar was bubbled into the buffer for $15 \mathrm{~min}$ before the measurement and continuously, gently, bubbled during the measurement. The obtained results in the SECM measurements clearly demonstrate the absence of any cathodic current related to the collection of $\mathrm{O}_{2}$ evolved from the sample. Furthermore, a considerable shift in the recorded current at the microelectrode toward more positive values was observed under irradiation and following a clear correlation between the sample current and the tip current under dark and light conditions (Fig. 5d). The shift in current at the SECM probe can then be attributed to the oxidation of the soluble mediator at the tip microelectrode that is polarized at a more negative potential than the redox peak observed for the mediator in previous experiments (Fig. 2c). Further measurements at a tip potential of $-200 \mathrm{mV}$ (vs. $\mathrm{Ag} / \mathrm{AgCl} / 3 \mathrm{M} \mathrm{KCl}$ ) indicate a reduction of the mediator in the light (Supplementary Fig. 6C). SECM measurements confirmed that the mediator diffuses from the iSyn through the solution to the tip. Thus, we can conclude that there is a water-soluble electron carrier that mediates between the iSyn and the electrode.

Finally, in an attempt to identify the electron carrier mediator if it is a metabolite, we carried out a metabolic profiling ${ }^{49,50}$ of Syn, iSyn, and iSyn that were illuminated in the BEPC. No significant change between the different samples profile was detected, suggesting that the gentle treatment does not cause a significant change in the amount of a metabolite but rather the release of the mediator to the medium or the outrace of the cells (Supplementary Fig. 7 and Supplementary Table 2).
DCMU has a unique effect in the iSyn BPEC. In the presence of DCMU, the maximum current is reached faster and the current is slightly enhanced (Fig. 2b, Supplementary Fig. 8A,B). However, the photocurrent lifetime is prolonged by the presence of added glucose in either the presence or absence of DCMU (Supplementary Fig. 8C), suggesting that DCMU affects the electron flow but not the stability of the photocurrent. Counter-intuitively, the presence of DCMU was found to be crucial for the photocurrent production by the iSyn $\triangle \mathrm{psbA}$, since in the absence of DCMU the photocurrent drops to $14 \%$ of the photocurrent obtained in the presence of DCMU (Supplementary Fig. 8B,D). Thus, even though iSyn $\Delta$ psbA lacks the well-studied PSII-binding-site of DCMU, the addition of the herbicide has a dramatic effect and actually was essential for the production of the $20-30 \mu \mathrm{A} \mathrm{cm}{ }^{-2}$ current obtained with iSyn $\triangle$ psbA even without DCMU (Supplementary Fig. $8 D$ ). The effect of DCMU in the iSyn $\Delta \mathrm{psbA}$ strongly indicates that DCMU has secondary binding sites with Synechocystis cells that have not yet been characterized. Moreover, DCMU increase the release of the mediator (Supplementary Fig. 8E) and as a results increases the photocurrent and decreases the time it takes to reach the maximum photocurrent (Fig. 2b, Supplementary Fig. 8C). Our results suggest that DCMU affects the production of the photocurrent in both iSyn and iSyn $\Delta$ psbA at secondary site(s) by either inhibiting forward electron flow within the respiratory system, or by enhancing the release of the mediator.

Hydrogen is produced in the BPEC. As no external redox components were added to the BPEC, we presumed that protons are reduced to molecular hydrogen at the Pt cathode. To measure hydrogen production, we built a gas-tight BPEC connected directly to a gas-chromatograph (GC) (Fig. 6a). The gas actively diffuses via ducts and is pumped into the GC, hence there is a short delay in the gas measurement. Figure $6 \mathrm{~b}$ shows the $\mathrm{H}_{2}(\mathrm{~g})$ production over time measured using this configuration applying a voltage of $650 \mathrm{mV}$ between the anode and cathode. The lack of $\mathrm{H}_{2}(\mathrm{~g})$ production in the absence of light and/or iSyn (Supplementary Table 1) indicates a correlation between the light-current and the $\mathrm{H}_{2}$ production. During the $2 \mathrm{~h}$ of illumination an average of $3.5 \mu \mathrm{mol} \mathrm{H}_{2(\mathrm{~g})} \mathrm{mg} \mathrm{Chl}^{-1} \mathrm{~h}^{-1}$ is produced at a Faradaic efficiency of ca. $36 \%$. The low Faradaic efficiency is most likely the result of molecular oxygen in the buffer, indicated by higher Faradaic efficiency in the absence of oxygen (Supplementary Fig. 9), or the mediator reduced on the cathode in addition to proton reduction.

In this study, we show light-driven current and hydrogen production with mildly treated live Synechocystis sp. PCC 6803 on a graphite electrode. The simple treatment makes the electron transfer possible from the cells to the electrode. This is the first time CV measurements indicate the existence of an endogenous mediator that can exit the cells' membranes in mildly treated live cyanobacteria $^{50,51}$. The electron extraction, by the intrinsic mediator, is photo-driven by PSI (Fig. 6c) that is reduced by the respiratory system, explaining the enhancing effect of glucose addition. In the absence of externally added glucose, the current originates from the internal carbohydrate pool produced during the growth phase. Whether PSI reduces the mediator directly or through other components of the photosynthetic electron transfer chain has not yet been ascertained. It is important to note that current is obtained from non-treated cells as well, however at much lower rates. This reiterates that the role of the treatment is to augment the amount of released mediator, and not the inducing of any modification to either of the two biological systems. During the review process of this article, a manuscript suggesting mediator dependent electron transfer in cyanobacteria 
a

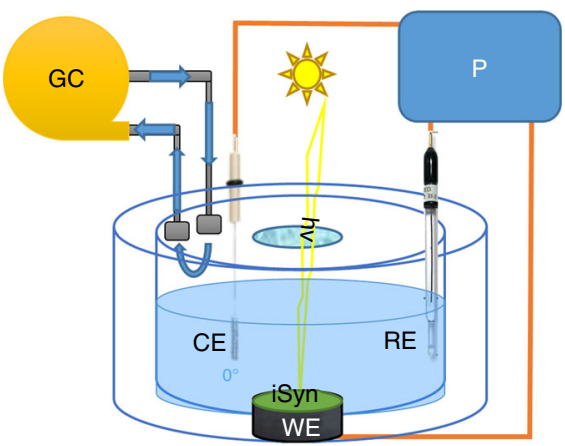

b

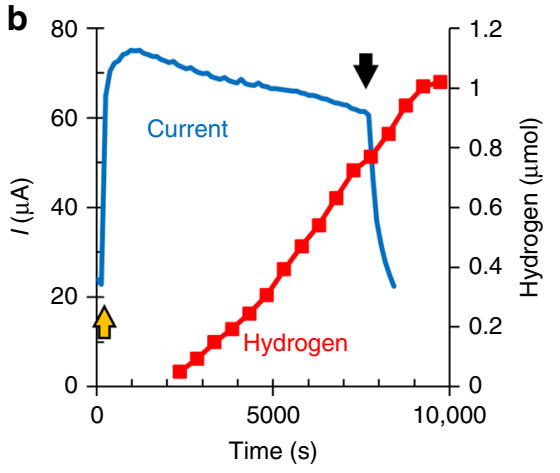

c

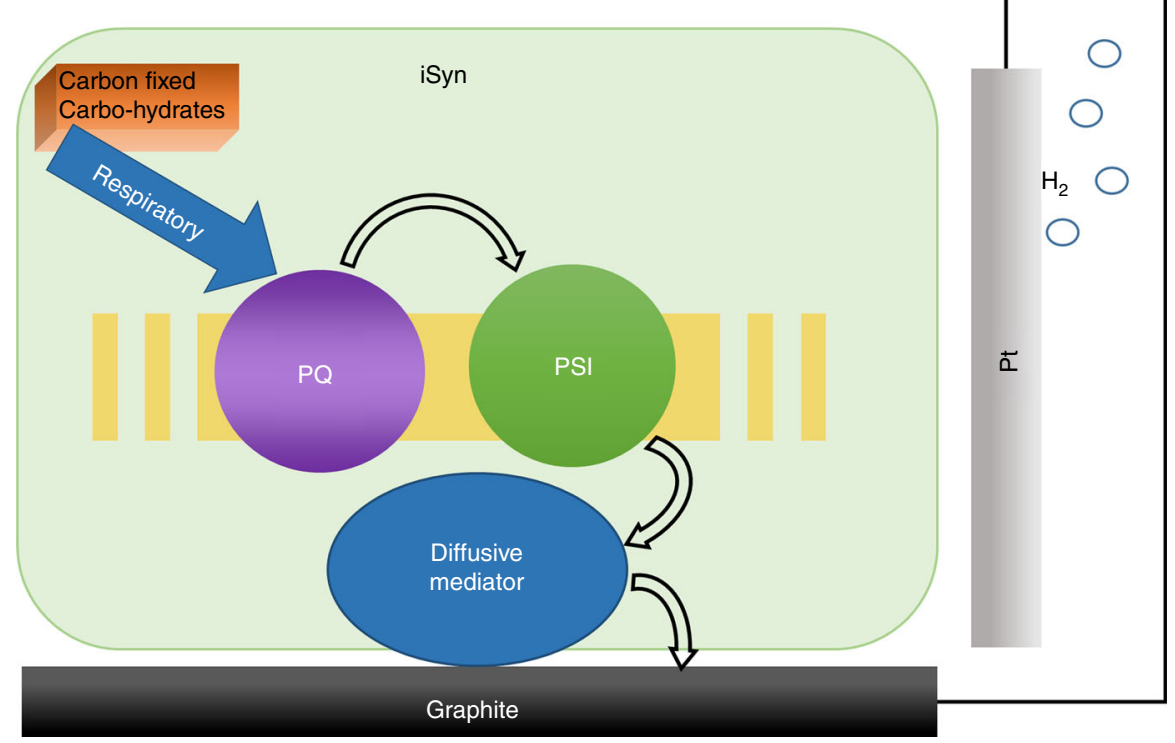

Fig. 6 Hydrogen is evolved on the cathode at a lower voltage than in water electrolysis. a Schematic drawing of the working setup for the hydrogen evolution measurements. The CA was measured at $50 \mathrm{mV}$ (vs. $\mathrm{Ag} / \mathrm{AgCl} / 3 \mathrm{M} \mathrm{NaCl}$ ) which corresponds to a voltage of $650 \mathrm{mV}$ between the anode and cathode. $\mathbf{b}$ Simultaneous CA measurement of photocurrent (blue) and GC measurement of hydrogen production (red) measured as a function of time for the iSyn at $50 \mathrm{mV}$ (vs. $\mathrm{Ag} / \mathrm{AgCl} / 3 \mathrm{M} \mathrm{NaCl}$ ). c Schematic drawing of the electron flow from carbohydrates (internal or external) via the respiratory, lightdriven activity of PSI, and an endogenous diffusive mediator to form hydrogen gas. The multiple electron transfer steps between the PQ and PSI are shown for simplicity as multiple curved arrows

was published. ${ }^{52,53}$ In this work, porous translucent electrodes were used in a BEPC cell containing Synechocystis cells to obtain electric currents that were higher than obtained in nonporous electrodes but significantly lower than reported in this work using iSyn and graphite electrode.

The use of iSyn instead of thylakoids may have the potential to utilize physiological repair mechanisms during the operation of the BPEC. This system, based on live Synechocystis and an endogenous mediator, can further be used to study extraction of electrons from live cells and with some future modifications can be used to generate photocurrent for a long time by cell rejuvenation.

In the recent past, utilization of biological systems for environmentally clean energy production has been offered as an attractive alternative to the use of fossil fuels. Our results presented here show that Cyanobacteria offer a unique opportunity to fully utilize the potential of biological energy conversion systems. We have shown here that in these live cells, electrons released during the reactions of the respiratory system can be "pumped" back to higher energy by the photosynthetic system, enabling both sustained current and the reduction of hydrogen at lower added bias. Indeed, coupled with other innovative uses of the solar spectrum ${ }^{24}$ we believe that the cyanobacterial system can serve a true paradigm for clean bio-energy production.

\section{Methods}

Preparation of mildly treated cells of Synechocystis. Cells of a late log phase culture $\left(\mathrm{OD}_{750}=1.0-1.5\right)$ that was grown as previously described in Larom et al. ${ }^{38,39}$ were centrifuged for $10 \mathrm{~min}$ at $15,200 \times \mathrm{g}$ in a SLA-1500 rotor and resuspended in Buffer A (20 mM Tris $\mathrm{pH} 8,400 \mathrm{mM} \mathrm{NaCl}$ and $\left.15 \mathrm{mM} \mathrm{MgCl}_{2}\right)$. The cells were then passed twice through a microfluidizer (Microfluidics Corporation) under a pressure of $10-15$ psi. The cells were centrifuged for $20 \mathrm{~min}$ at $27,660 \times g$ and the cell pellet was resuspended in $100 \mathrm{mM}$ phosphate buffer ( $\mathrm{pH} \mathrm{6}$ ). The final concentration of chl $a$ was determined in $80 \%(\mathrm{v} / \mathrm{v})$ acetone according to Arnon $^{53,54}$. The final chlorophyll concentration of all samples was $0.6-1.3 \mathrm{mg}$ chl $\mathrm{ml}^{-1}$.

Preparation of all other Synechocystis samples. Thylakoid membranes (mSyn) were isolated in a fashion similar to the iSyn preparation; however, a French pressure cell (Sim Aminco Spectronic Instruments) operating at 1500 psi was used instead of the microfluidizer, followed by centrifugation for $2 \mathrm{~min}$ at $600 \times \mathrm{g}$ in order to pellet unbroken cells and 20 min centrifugation at $27,660 \times g$ to pellet the thylakoids. For $\mathrm{mSyn} \Delta \mathrm{psbA}$ preparation, we used the microfluidizer at high pressure of 90 psi circulating for 28 pulses. Untreated cells (Syn) were pelleted and resuspended in phosphat buffer ( $\mathrm{pH}$ 6). Osmotic shocked cells (OsSyn) were resuspended in buffer $\mathrm{A}$ and pelleted after $20 \mathrm{~min}$ and resuspended in phosphate buffer ( $\mathrm{pH} \mathrm{6)}$. The cells treated with different pressures were prepared similar to iSyn, but under higher pressure in the microfluidizer step. The final concentration of all samples was $0.6-1.3 \mathrm{mg} \mathrm{chl} \mathrm{ml}^{-1}$.

Electrochemical measurements. Electrochemical measurements were performed either at the Solar Simulation Lab (Viterbi Faculty of Electrical Engineering, 
Technion) using a Zennium electrochemical workstation (Zahner Elektrik) and a solar simulator light source (Oriel Sol3A), or at the Hydrogen Lab (Grand Technion Energy Program) with an nStat multichannel potentiostat (Ivium) and a Xenon lamp solar simulator (Abet). For some measurements, we used a Palmsens3 (Palmsens) potentiostat and A1 light line (Sciencetech). All measurements were carried out in a three electrodes configuration under the illumination of one solar unit (1SU). Graphite (Graphite Store, pn\#bl001230) was used as a working electrode, a Pt electrode (ALS) served as the counter electrode and a $\mathrm{Ag} / \mathrm{AgCl} / 3 \mathrm{M}$ $\mathrm{NaCl}$ as a reference electrode (ALS). For each measurement, a sample containing $150 \mu \mathrm{g}$ of chl $a$ was placed on the $1.8 \mathrm{~cm}$ diameter graphite electrode. The sample was not stirred or replaced during the measurement. The medium was phosphate buffer (100 mM phosphate, $\mathrm{pH}$ 6) containing $100 \mathrm{mM} \mathrm{NaCl}$. When indicated, 150 $\mu \mathrm{M}$ of DCMU was added. For the $\Delta \mathrm{psbA}$ mutant, chlorophyll amount of $50 \mu \mathrm{g}$ was used. For the relevant electrochemical measurements, $6 \mathrm{mM}$ of D-glucose in the absence or presence of $5 \mathrm{mM}$ iodoacetic acid (sodium salt) were added.

Quantum efficiency measurements. The EQE of the BPEC containing iSyn (150 $\mu \mathrm{g} \mathrm{chl}$ ) was measured at a potential of $150 \mathrm{mV}$ (vs. $\mathrm{Ag} / \mathrm{AgCl} / 3 \mathrm{M} \mathrm{NaCl}$ ). The iSyn cells were illuminated with monochromatic light obtained by coupling a white light source to a monochromator (Cornerstone CS260) with a spectral bandwidth of 10 $\mathrm{nm}$. Fresh cells were used in each measurement, and the maximum photocurrent was recorded. The photon flux was obtained from the light intensity measured by a power meter (918D High Performance Photodiode Sensor), and the EQE was calculated according to equation 1 :

$$
\mathrm{EQE}=\frac{\text { Maximum photocurrent } / q}{\text { Photon flux }}
$$

where $q$ is the electron charge.

When analyzing iSyn $\Delta \mathrm{psbA}$ we used $50 \mu \mathrm{g}$ chl for each measurement and the bandwidth was $20 \mathrm{~nm}$.

Oxygen evolution measurements. For measurement the photosynthetic activity by analyzing the rate of oxygen evolution, the cells $(10 \mu \mathrm{g} \mathrm{chl} \mathrm{a)} \mathrm{were} \mathrm{placed} \mathrm{in}$ phosphate buffer ( $\mathrm{pH}$ 6) in a final volume of $1 \mathrm{ml}$. A volume of $3 \mathrm{mM}$ 2,6-dichloro1,4-benzoquinone (DCBQ) was used as an electron acceptor and oxygen evolution was determined using a Clark-type electrode (Hansatech). Following preincubation in the dark for $2 \mathrm{~min}$, the cells were illuminated with a white light halogen projector lamp $(M R C)$ for $1 \mathrm{~min}$ and the increase in oxygen concentration was digitally measured.

Gas chromatography experiments. Hydrogen measurements were performed in the Hydrogen Lab (Grad Technion Energy Program) using an Agilent 7890 A GC system (Agilent technologies) with a molsieve $5 \AA$ column, $10 \mathrm{ft}{ }^{\star} 1 / 8^{\prime \prime} * 2 \mathrm{~mm}$ (Agilent technologies) to determine $\mathrm{H}_{2}$ production. Gas from the BPEC was continuously pumped to the GC. The applied potential on the working electrode was $50 \mathrm{mV}$ (vs. $\mathrm{Ag} / \mathrm{AgCl} / 3 \mathrm{M} \mathrm{NaCl}$ ). The bias between the anode and cathode measured by a voltmeter (m832, Mastech) was $650 \mathrm{mV}$. Calibration was performed by measuring known amounts of hydrogen directly injected to the GC.

Scanning electron microscopy. The morphology of cellular samples was characterized by high-resolution scanning electron microscopy (SEM) (Ultra PLUS, Zeiss). Further experimental details are found in the figure legend.

Purification of the mediator. iSyn cells, correspond to $400 \mu \mathrm{g}$ chlorophyll from the stock solution, were illuminated for $5 \mathrm{~min}$ (without DCMU) in a $1.5 \mathrm{ml}$ tube and then centrifuged (5417 R, Eppendorf) for $1 \mathrm{~min}$ at max speed. The supernatant was collected, the pellet was resuspended in $250 \mu$ l Phosphate buffer, centrifuged again, and the supernatant was collected. Filtration was performed using $3 \mathrm{kD}$ (Vivaspin 500, Sartorius Stedim biotech) and centrifugation (5417 R, Eppendorf) for $1 \mathrm{~h}$ at $14,000 \times g$. Heat inactivation was performed in a block heater (SKS Biomedical instruments) at $60^{\circ} \mathrm{C}$ for $10 \mathrm{~min}$

Scanning electrochemical microscopy. SECM experiments were performed using a $\mathrm{Ag} / \mathrm{AgCl} / 3 \mathrm{M} \mathrm{KCl}$ double junction reference electrode, a Pt cylindrical mesh as counter electrode, and a BOD modified carbon microelectrode as electrochemical probe. The BOD modified tip was fabricated following a previously reported procedure $^{54,55}$ by modifying a carbon-based microelectrode with a mixture of BOD embedded in an Os-complex modified polymer matrix ${ }^{47,48}$. The SECM setup is schematically shown in Fig. $5 c$ and is based on a previously described system ${ }^{46,47}$ consisting of three stepmotor driven micrometer screws (Owis) for positioning of the SECM tip in $x$-, $y$-, and $z$-directions, a bipotentiostat (PGU-BI 100, Jaissle), and a control software programmed using Visual Basic 6.0. A 150 W Hg-Xe lamp (LC8, Hamamatsu photonics) was used for irradiation of the sample at an incident light intensity of $55 \mathrm{~mW} \mathrm{~cm}^{-2}$ (visible light). The sample was placed in a Teflon SECM cell sealed with an O-ring exposing an area of about $0.385 \mathrm{~cm}^{2}$. The tip was positioned in close proximity $(\sim 30 \mu \mathrm{m})$ to the analyzed sample by recording $\mathrm{z}$ approach curves in air-saturated buffer before adding the iSyn. The BOD modified tip was polarized at 0 or $-200 \mathrm{mV}$ (vs. $\mathrm{Ag} / \mathrm{AgCl} / 3 \mathrm{M} \mathrm{KCl}$ ). During the measurement, a given potential of $150 \mathrm{mV}$ (vs. $\mathrm{Ag} / \mathrm{AgCl} / 3 \mathrm{M} \mathrm{KCl}$ ) was applied to the sample. Both the tip and sample current were recorded during light and dark periods. For the SECM measurements, we used frozen/thawed iSyn cells. After preparation of the cells as described above, dimethyl sulfoxide was added to the cells, prior to flash-freezing in liquid nitrogen. iSyn was later thawed on ice and used for the SECM experiments.

Procedures for PBS spectroscopic measurements, confocal imaging, Tris treatment, colony formation test, and metablomics profiling were detailed in Supplementary Methods.

Data availability. All data presented in this paper can be obtained from the corresponding authors upon reasonable request.

Received: 15 July 2017 Accepted: 4 May 2018

Published online: 04 June 2018

\section{References}

1. McCormick, A. J. et al. Photosynthetic biofilms in pure culture harness solar energy in a mediatorless bio-photovoltaic cell (BPV) system. Energy Environ. Sci. 4, 4699-4709 (2011).

2. Kaiser, B. K. et al. Fatty aldehydes in cyanobacteria are a metabolically flexible precursor for a diversity of biofuel products. PLoS ONE 8, e58307 (2013).

3. Deng, M.-D. \& Coleman, J. R. Ethanol synthesis by genetic engineering in cyanobacteria. Appl. Environ. Microbiol. 65, 523-528 (1999).

4. Whitton, B. A. Ecology of Cyanobacteria II: Their Diversity in Space and Time. (Springer, Germany, 2012).

5. Szewczyk, S., Giera, W., D’Haene, S., van Grondelle, R. \& Gibasiewicz, K. Comparison of excitation energy transfer in cyanobacterial photosystem I in solution and immobilized on conducting glass. Photosynth. Res. 132, 111-126 (2017).

6. Novoderezhkin, V. I. et al. Mixing of exciton and charge-transfer states in light-harvesting complex Lhca4. Phys. Chem. Chem. Phys. 18, 19368-19377 (2016).

7. Gobets, B., van Stokkum, I. H. M., van Mourik, F., Dekker, J. P. \& van Grondelle, R. Excitation wavelength dependence of the fluorescence kinetics in photosystem I particles from Synechocystis PCC 6803 and Synechococcus elongatus. Biophys. J. 85, 3883-3898 (2003).

8. Brecht, M., Radics, V., Nieder, J. B., Studier, H. \& Bittl, R. Red antenna states of photosystem I from Synechocystis PCC 6803. Biochemistry 47, 5536-5543 (2008).

9. Adir, N. Elucidation of the molecular structures of components of the phycobilisome: Reconstructing a giant. Photosynth. Res. 85, 15-32 (2005).

10. van Amerongen, H. \& Croce, R. Light harvesting in photosystem II. Photosynth. Res. 116, 251-263 (2013).

11. Croce, R. \& van Amerongen, H. Light-harvesting in photosystem I. Photosynth. Res. 116, 153-166 (2013)

12. Nelson, N. \& Yocum, C. F. Structure and function of photosystems I and II. Annual Review of Plant Biology 57, 521-565 (2006).

13. Raven, J. A. \& Beardall, J. In: P. A. del Giorgio, P. JleB. Williams Ed. by. Respiration in aquatic photolithotrophs. Respir. Aquat. Ecosyst (pp. 36-46. Oxford Univ. Press, New York, 2005).

14. Vermaas, W. F. J. Photosynthesis and respiration in cyanobacteria. Encycl. Life Sci. 245-251 (2001)

15. Osanai, T. et al. Genetic engineering of group $2 \sigma$ factor SigE widely activates expressions of sugar catabolic genes in Synechocystis species PCC 6803. J. Biol. Chem. 286, 30962-30971 (2011).

16. Knoop, H. et al. Flux balance analysis of cyanobacterial metabolism: the metabolic network of Synechocystis sp. PCC 6803. PLoS Comput. Biol. 9 , e1003081 (2013).

17. Bradley, R. W., Bombelli, P., Lea-Smith, D. J. \& Howe, C. J. Terminal oxidase mutants of the cyanobacterium Synechocystis sp. PCC 6803 show increased electrogenic activity in biological photo-voltaic systems. Phys. Chem. Chem. Phys. 15, 13611-13618 (2013).

18. Lea-Smith, D. J., Bombelli, P., Vasudevan, R. \& Howe, C. J. Photosynthetic, respiratory and extracellular electron transport pathways in cyanobacteria. Biochim. Biophys. Acta (BBA) Bioenerg. 1857, 247-255 (2016).

19. McCormick, A. J. et al. Biophotovoltaics: oxygenic photosynthetic organisms in the world of bioelectrochemical systems. Energy Environ. Sci. 8, 1092-1109 (2015).

20. Zhang, L. et al. Deletion of Synechocystis sp. PCC 6803 leader peptidase LepB1 affects photosynthetic complexes and respiration. Mol. Cell. Proteom. 12, 1192-1203 (2013)

21. Schmetterer, G., Alge, D. \& Gregor, W. Deletion of cytochrome c oxidase genes from the cyanobacterium Synechocystis sp. PCC6803: Evidence for alternative respiratory pathways. Photosynth. Res. 42, 43-50 (1994). 
22. Larom, S. et al. The Photosystem II D1-K238E mutation enhances electrical current production using cyanobacterial thylakoid membranes in a biophotoelectrochemical cell. Photosynth. Res. 126, 161-169 (2015).

23. Pinhassi, R. I. et al. Hybrid bio-photo-electro-chemical cells for solar water splitting. Nat. Commun. 7, 12552 (2016).

24. Zhao, F. et al. Light induced $\mathrm{H} 2$ evolution from a biophotocathode based on photosystem 1-Pt nanoparticles complexes integrated in solvated redox polymers films. J. Phys. Chem. B 119, 13726-13731 (2015).

25. Efrati, A. et al. Assembly of photo-bioelectrochemical cells using photosystem I-functionalized electrodes. Nat. Energy 1, 15021 (2016).

26. Gizzie, E. A. et al. Photosystem I-polyaniline/TiO 2 solid-state solar cells: simple devices for biohybrid solar energy conversion. Energy Environ. Sci. 8, 3572-3576 (2015).

27. Sekar, N., Umasankar, Y. \& Ramasamy, R. P. Photocurrent generation by immobilized cyanobacteria via direct electron transport in photobioelectrochemical cells. Phys. Chem. Chem. Phys. 16, 7862 (2014).

28. Sekar, N., Jain, R., Yan, Y. \& Ramasamy, R. P. Enhanced photobioelectrochemical energy conversion by genetically engineered cyanobacteria. Biotechnol. Bioeng. 113, 675-679 (2016).

29. Sawa, M. et al. Electricity generation from digitally printed cyanobacteria. Nat. Commun. 8, 1327 (2017)

30. Mershin, A. et al. Self-assembled photosystem-I biophotovoltaics on nanostructured $\mathrm{TiO} 2$ and $\mathrm{ZnO}$. Sci. Rep. 2, 234 (2012).

31. Yehezkeli, O. et al. Integrated photosystem II-based photo-bioelectrochemical cells. Nat. Commun. 3, 742 (2012).

32. Efrati, A., Tel-Vered, R., Michaeli, D., Nechushtai, R. \& Willner, I. Cytochrome c-coupled photosystem I and photosystem II (PSI/PSII) photobioelectrochemical cells. Energy Environ. Sci. 6, 2950-2956 (2013).

33. Li, J. et al. Integrating photosystem II into a porous TiO 2 nanotube network toward highly efficient photo-bioelectrochemical cells. J. Mater. Chem. A 4, 12197-12204 (2016)

34. Zhao, F., Sliozberg, K., Rögner, M., Plumeré, N. \& Schuhmann, W. The role of hydrophobicity of Os-complex-modified polymers for photosystem 1 based photocathodes. J. Electrochem. Soc. 161, H3035-H3041 (2014).

35. Yao, D. C. I., Brune, D. C. \& Vermaas, W. F. J. Lifetimes of photosystem I and II proteins in the cyanobacterium Synechocystis sp. PCC 6803. FEBS Lett. 586, 169-173 (2012).

36. McCormick, A. J. et al. Hydrogen production through oxygenic photosynthesis using the cyanobacterium Synechocystis sp. PCC 6803 in a biophotoelectrolysis cell (BPE) system. Energy Environ. Sci. 6, 2682-2690 (2013).

37. Pinhassi, R. I. et al. Photosynthetic Membranes of Synechocystis or Plants Convert Sunlight to Photocurrent through Different Pathways due to Different Architectures. PLoS ONE 10, e0122616 (2015).

38. Larom, S., Salama, F., Schuster, G. \& Adir, N. Engineering of an alternative electron transfer path in photosystem II. Proc. Natl Acad. Sci. USA 107 9650-9655 (2010).

39. Calkins, J. O., Umasankar, Y., O’Neill, H. \& Ramasamy, R. P. High photoelectrochemical activity of thylakoid-carbon nanotube composites for photosynthetic energy conversion. Energy Environ. Sci. 6, 1891-1900 (2013).

40. Cereda, A. et al. A bioelectrochemical approach to characterize extracellular electron transfer by Synechocystis sp. PCC6803. PLoS ONE 9, e91484 (2014).

41. Wei, X., Lee, H. \& Choi, S. Biopower generation in a microfluidic bio-solar panel. Sens. Actuators B Chem. 228, 151-155 (2016).

42. Yruela, I., Montoya, G., Alonso, P. J. \& Picorel, R. Identification of the pheophytin-QA-Fe domain of the reducing side of the photosystem II as the Cu (II)-inhibitory binding site. J. Biol. Chem. 266, 22847-22850 (1991).

43. Nixon, P. J., Metz, J. G., Roegner, M. \& Diner, B. A. A Synechocystis PCC $6803 p s b A$ deletion mutant and its Transformation with a $p s b A$ gene from a higher plant. in Current research in photosynthesis 471-474 (Springer, Germany, 1990).

44. Marsili, E. et al. Shewanella secretes flavins that mediate extracellular electron transfer. Proc. Natl Acad. Sci. USA 105, 3968-3973 (2008).

45. Essmann, V. et al. In operando investigation of electrically coupling of photosystem 1 and photosystem 2 by means of bipolar electrochemistry. Anal. Chem. 89, 7160-7165 (2017)

46. Conzuelo, F. et al. High-resolution analysis of photoanodes for water splitting by means of scanning photoelectrochemical microscopy. Anal. Chem. 89, 1222-1228 (2017).

47. Badura, A. et al. Photo-induced electron transfer between photosystem 2 via cross-linked redox hydrogels. Electroanalysis 20, 1043-1047 (2008)

48. Sokol, K. P. et al. Rational wiring of photosystem II to hierarchical indium tin oxide electrodes using redox polymers. Energy Environ. Sci. 9, 3698-3709 (2016).

49. Treves, H. et al. Metabolic flexibility underpins growth capabilities of the fastest growing alga. Curr. Biol. 27, 2559-2567 (2017). e3.

50. Schuergers, N., Werlang, C., Ajo-Franklin, C. M. \& Boghossian, A. A. A synthetic biology approach to engineering living photovoltaics. Energy Environ. Sci. 10, 1102-1115 (2017).
51. Zou, Y., Pisciotta, J. \& Baskakov, I. V. Nanostructured polypyrrole-coated anode for sun-powered microbial fuel cells. Bioelectrochemistry 79, 50-56 (2010).

52. Wenzel, T., Härtter, D., Bombelli, P., Howe, C. J. \& Steiner, U. Porous translucent electrodes enhance current generation from photosynthetic biofilms. Nat. Commun. 9, 1299 (2018).

53. Arnon, D. I. Copper enzymes in isolated chloroplasts. Polyphenoloxidase in Beta vulgaris. Plant Physiol. 24, 1 (1949).

54. Zhao, F. et al. A novel versatile microbiosensor for local hydrogen detection by means of scanning photoelectrochemical microscopy. Biosens. Bioelectron. 94, 433-437 (2017).

55. Chabi, S. et al. Membranes for artificial photosynthesis. Energy Environ. Sci. 10, 1320-1338 (2017).

\section{Acknowledgements}

This work was funded by the I-CORE Program of the Planning and Budgeting Committee, the Israel Science Foundation (Grant No. 152/11), a grant from the transformative program of the US-Israel Binational Science Foundation (BSF grant No. 2011556), and a grant from the Deutsche Forschungsgemeinschaft (DIP project LU315/ 17-1). The results reported in this work were obtained using central facilities at the Technion's Photovoltaic Laboratory, supported by the Nancy \& Stephen Grand Technion Energy Program (GTEP) and by the Russell Berrie Nanotechnology Institute (RBNI), and at the Technion's Hydrogen Technologies Research Laboratory (HTRL), supported by the Adelis Foundation and by the Solar Fuels I-CORE. We thank Dr. Anatoly Bekkerman and Semion Goberman the Faculty of Chemistry technical team for constructing the photocell; Dr. Guy Ankonina from the PV lab for assistance in design and execution of electrochemical tests; Dr. Nurit Waldman and Dr. Shany Hershkovitz from the hydrogen lab for assistance in quantification the hydrogen production. We thank Asaf Kay for help with the SEM images. We thank Yaniv Shlosberg, Shmuel Carmeli, and Larisa Panz for their assistance in analysis of the mediator. D.K. and G.Sa. are supported by fellowships of the Nancy \& Stephen Grand Technion Energy Program (GTEP). D.K. is supported by fellowships of the Israeli Ministry of Science, Technology and Space. G.Sa. is supported by a Schulich Graduate fellowship.

\section{Author contributions}

G.Sa., D.K., A.R., G.Sc. and N.A. conceived the idea. D.K., G.Sa., A.R., G.Sc. and N.A. designed the experiments. G.Sa. and D.K performed the main experiments. G.Sa, F.C. and F.Z. performed the SECM experiments. T.T. performed part of the cell viability experiments. V.L. performed the immunoblot experiment, S.M., J. S. and A.A. performed metabolomics profiling. A.R., N.A., G.Sc., W.S., F.C., F.Z., G.Sa. and D.K. analyzed the data. A.R., N.A., W.S., F.C, G.Sc., G.Sa. and D.K. wrote the paper. A.R., N.A. and G.Sc. supervised the entire research.

\section{Additional information}

Supplementary Information accompanies this paper at https://doi.org/10.1038/s41467 018-04613-x.

Competing interests: The authors declare no competing interests.

Reprints and permission information is available online at http://npg.nature.com/ reprintsandpermissions/

Publisher's note: Springer Nature remains neutral with regard to jurisdictional claims in published maps and institutional affiliations.

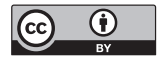

Open Access This article is licensed under a Creative Commons Attribution 4.0 International License, which permits use, sharing, adaptation, distribution and reproduction in any medium or format, as long as you give appropriate credit to the original author(s) and the source, provide a link to the Creative Commons license, and indicate if changes were made. The images or other third party material in this article are included in the article's Creative Commons license, unless indicated otherwise in a credit line to the material. If material is not included in the article's Creative Commons license and your intended use is not permitted by statutory regulation or exceeds the permitted use, you will need to obtain permission directly from the copyright holder. To view a copy of this license, visit http://creativecommons.org/ licenses/by/4.0/.

(c) The Author(s) 2018 